\title{
On Concurrent Solutions in Differential Games
}

\author{
Romar Correa \\ University of Mumbai, \\ Department of Economics, \\ Vidyanagari, Mumbai 400 098, India \\ romarcorrea10@economics.mu.ac.in
}

\begin{abstract}
We examine solutions in which neither player is worse off from the leadership of one in a policy maker-public game. The loop model of dynamic games is used. Outcome space is dotted with equivalence classes of solutions. The Dynamic Stochastic General Equilibrium (DSGE) results and their New Keynesian variants might represent one category. The economy is the neighborhood of a market-clearing equilibrium with Pareto-optimal properties modulo frictions. Our interest lies in the 'old' Keynesian genus where the representative state is one of involuntary unemployment. Two information sets are relevant. In the first case, agents look to the past and the present. In the second, they are bound by the information provided in the present. The standard analysis pertains to DSGE models under full information. We show, in contrast, that in a situation of structural disequilibrium and feedback information, all parties are better off reneging on the social compact to achieve a superior class of solutions.
\end{abstract}

Keywords: information structures, time consistency, credibility and reputation

Received 21 January 2011

Revised 21 April 2011

Accepted 1 May 2011

JEL Classification: C73, E61

\section{Introduction}

The limitations of the mainstream dynamic stochastic general equilibrium (DSGE) model have been advertised in recent times because of its inability to model financial-real breakdowns. Our systems appraisal below rests on the following assumptions. While the foundations of the DSGE model are often proclaimed to be Walrasian, scholars have questioned this claim (Kirman, 2010). The dynamical equation of Definition 1 is intended to capture the idea that a plethora of disequilibrium adjustment mechanisms may be available. Trade takes place sequentially. At each stage, the state of the world is available for observation. The economy, in turn, is the outcome of the actions of agents. Put differently, agents act in a social context. As the context changes, preferences may be influenced. Secondly, the fundamental notion of 'representation of strategies along trajectories' below is another way of expressing 'model uncertainty' (Dow et. al., 2009). No single model can capture the feedback between individual and system and policy. In a situation of uncertainty as distinct from risk, the probability distributions of shocks remain unknown. Without a given model, the disturbance terms cannot be expressed formally. The assumption of rational expectations does not help as there is no benchmark model to anchor expectations.

\section{Preliminaries}

A corollary to DSGE theorems is the inefficiency role played by the authorities. The government, in the form of the central bank, is the leader and firms and workers are followers in the macroeconomic game. The following account is drawn from Başar and Jan Olsder (1995). Hierarchical or Stackelberg equilibria are an important component of the loop model of dynamic games. There are two possibilities. In the prior commitment mode of play decisions are made at the outset. Feedback games, on the other hand, are of the delayed commitment type. Each player waits to find out the current value of the state and only then announces her action.

Time inconsistency emerges in prior commitment decisions. In the absence of formal or informal precepts, in every period there is an incentive for the leader to renege on her commitment. In the macroeconomic illustrations the payoffs to the government by 'cheating' thereby increase and the payoffs to the private sector fall, both being calculated in relation to the (Nash) payoffs that would obtain in a situation where the players were symmetrically positioned. The prior commitment strategy is nonconcurrent. Since the inconsistency arises because of the information that is forthcoming in every period, the recommended solution to the problem is to employ open-loop strategies, that is, 
strategies that depend only on the initial state and time. In terms of the debate in macroeconomics, the case is made for rules as against discretion. Our understanding of leadership in the policy game is the opposite (Fischbocher, 2010). The leader is not an outsider. Leading by example she induces others to follow suit and act in the collective interest. Furthermore, typically her discount rate would need to be different from those of the followers. For instance, she might need to be more patient and even need to have other-regarding preferences.

In the agent-based computational view, the acquisition and use of information are central concepts (Tubaro, 2009). Induction scores over deduction. The loop model of dynamic games utilizes a finer classification of information structures than that which informs the discussion in economics. It will be shown that under dynamic information, both the followers as well as the leader are not worse off playing the Stackelberg game than the associated Nash game. In addition, subsets of dynamic information patterns will be identified wherein the Feedback Stackelberg Solution is 1. time-inconsistent and 2. both time-consistent and optimal.

Definition 1. A two-person discrete-time deterministic infinite dynamic game of fixed duration involves:

(i) An index set $\mathbf{K}=\{1, \ldots, K\}$ denoting the stages of the game, where $K$ is the maximum number of moves a player is allowed to make.

(ii) An infinite set $X$ with some topological structure called the state space of the game, to which the state of the game $x_{k}$ belongs for all $k \in \mathbf{K} \cup\{K+1\}$.

(iii) An infinite set $U_{k}^{i}$ with some topological structure defined for each stage $k$ and player $i$ called the action set of $\mathbf{P} i$ at stage $k$. Its elements are permissible actions $u_{k}^{i}$ of $\mathbf{P} i$ at stage $k$.

(iv) A function $\mathrm{f}_{\mathrm{k}}: \mathrm{X} \times \mathrm{U}_{\mathrm{k}}^{1} \times \mathrm{U}_{\mathrm{k}}^{2} \rightarrow \mathrm{X}$, defined for each stage $k$, so that

$$
\mathrm{x}_{\mathrm{k}+1}=\mathrm{f}_{\mathrm{k}}\left(\mathrm{x}_{\mathrm{k}}, \mathrm{u}_{\mathrm{k}}^{1}, \mathrm{u}_{\mathrm{k}}^{2}\right), k \in \mathbf{K}
$$

for some $x_{1} \in X$ called the initial state of the game. The difference equation above is called the state equation of the dynamic game.

(v) A finite set $\eta_{k}^{i}$ defined for each stage $k$ and player $i$ as a subcollection of $\left\{x_{1}, \ldots, x_{k} ; u_{1}^{1}, \ldots, u_{k-1}^{1} ; u_{1}^{2}, \ldots, u_{k-1}^{2}\right\}$ which determines the information gained and recalled by $\mathbf{P} i$ at stage $k$. Specification of $\eta_{k}^{i}$ for all stages $k$ characterizes the information structure of $\mathbf{P} i$ and the collection over all players of these information structures is the information structure of the game.

(vi) $\quad$ s set $\mathrm{N}_{\mathrm{k}}^{\mathrm{i}}$ defined for each stage $k$ and player $i$ as an appropriate subset of $\left\{(X \times \ldots X) \times\left(U_{1}^{1} \times \ldots U_{k-1}^{1}\right) \times\left(U_{1}^{2} \times \ldots U_{k-1}^{2}\right)\right\}$ compatible with $\eta_{k}^{i} \cdot N_{k}^{i}$ is called the information space of $\mathbf{P} i$ at stage $k$.

(vii) A prespecified class $\Gamma_{\mathrm{k}}^{\mathrm{i}}$ of mappings $\gamma_{\mathrm{k}}^{\mathrm{i}}: \mathrm{N}_{\mathrm{k}}^{\mathrm{i}} \rightarrow \mathrm{U}_{\mathrm{k}}^{\mathrm{i}}$ which are the permissible strategies of $\mathbf{P} i$ at stage $k$. The aggregate mapping $\gamma^{i}=\left\{\gamma_{1}^{i}, \gamma_{2}^{i}, \ldots, \gamma_{k}^{i}\right\}$ is a strategy for $\mathbf{P} i$ in the game and the class $\Gamma^{i}$ of all such mappings $\gamma^{\prime}$ so that $\gamma_{\mathrm{k}}^{\mathrm{i}} \in \Gamma_{\mathrm{k}^{\prime}}^{\mathrm{i}} \mathrm{k} \in \mathrm{K}$, is the strategy space of $\mathbf{P}$.

(viii) A functional $\mathrm{J}^{\mathrm{i}}:\left(\mathrm{X} \times \mathrm{U}_{1}^{1} \times \mathrm{U}_{1}^{2}\right) \times \ldots \times\left(\mathrm{X} \times \mathrm{U}_{\mathrm{K}}^{1} \times \mathrm{U}_{\mathrm{K}}^{2}\right) \rightarrow \mathrm{R}$, defined for each player and called the cost functional of $\mathbf{P}$ in the game. The cost functional is said to be stage-additive if there exists $g_{\mathrm{k}}^{\mathrm{i}}: \mathrm{X} \times \mathrm{X} \times \mathrm{U}_{\mathrm{k}}^{1} \times \mathrm{U}_{\mathrm{k}}^{2} \rightarrow \mathrm{R},(k \in \mathbf{K})$, so that

where

$$
J^{i}\left(u^{1}, u^{2}\right)=\sum_{k=1}^{k} g_{k}^{i}\left(x_{k+1}, u_{k}^{1}, u_{k}^{2}, x_{k}\right),
$$

$$
u^{j}=\left(u_{1}^{j}, u_{k}^{j}\right)
$$

The information structures of relevance are as follows: 
Definition 2. Pi's information structure is said to be open-loop (OL) if $\eta_{\mathrm{k}}^{\mathrm{i}}=\left\{\mathrm{x}_{1}\right\}, k \in \mathbf{K}$, closed-loop perfect state (CLPS) if $\eta_{\mathrm{k}}^{\mathrm{i}}=\left\{\mathrm{x}_{1}, \ldots, \mathrm{x}_{\mathrm{k}}\right\}, k \in \mathbf{K}$, memoryless perfect state (MPS) if $\eta_{\mathrm{k}}^{\mathrm{i}}=\left\{\mathrm{x}_{1}, \mathrm{x}_{\mathrm{k}}\right\}, k \in \mathbf{K}$, feedback perfect state (FB) if $\eta_{k}^{i}=\left\{x_{k}\right\}, k \in \mathbf{K}$.

The Noncooperative (Nash) Equilibrium Solution is given by

Definition 3. A pair of strategies $\left\{\gamma^{1^{*}}, \gamma^{2^{*}}\right\}$ with $\gamma^{i^{*}} \in \Gamma^{\mathrm{i}} ; i=1,2$, is said to constitute a Nash Equilibrium Solution if, and only if, the following inequalities are satisfied for all $\gamma^{i} \in \Gamma^{i}, i=1,2$.

$$
\begin{aligned}
& J^{2^{*}} \cong J^{1}\left(\gamma^{1^{*}}, \gamma^{2^{*}}\right) \leq J^{1}\left(\gamma^{1}, \gamma^{2^{*}}\right) \\
& J^{2^{*}} \cong J^{2}\left(\gamma^{1^{*}}, \gamma^{2^{*}}\right) \leq J^{2}\left(\gamma^{1^{*}}, \gamma^{2}\right)
\end{aligned}
$$

In the next section we restrict our attention to feedback games where at the time of her act each player has perfect information concerning the current level of play. In that case, the set of inequalities above is rewritten as

Definition 4. A pair of strategies $\left\{\gamma^{i^{*}}, \gamma^{2^{*}}\right\}$ constitutes a Feedback Nash Equilibrium Solution (FNE) if it satisfies the following inequalities for all $\gamma_{k}^{i} \in \Gamma_{k}^{i}, i=1,2, k \in \mathbf{K}$.

$$
\begin{aligned}
& \mathrm{J}^{1^{*}} \leq \mathrm{J}^{1}\left(\gamma_{1}^{1}, \ldots, \gamma_{\mathrm{K}}^{1} ; \gamma^{2^{*}}\right) \\
& \mathrm{J}^{2^{*}} \leq \mathrm{J}^{2}\left(\gamma^{1^{*}} ; \gamma_{1}^{2}, \ldots, \gamma_{\mathrm{K}}^{2}\right)
\end{aligned}
$$

On any pair of Nash equilibrium strategies that satisfies the above inequalities, impose the further restriction that it satisfies the following $K$ inequalities:

At stage $K$

$$
\begin{aligned}
& J^{1}\left(\gamma_{1}^{1}, \ldots, \gamma_{\mathrm{K}-1}^{1}, \gamma_{\mathrm{K}}^{1 *} ; \gamma_{1}^{2}, \ldots, \gamma_{\mathrm{K}-1}^{2}, \gamma_{\mathrm{K}}^{2^{*}}\right) \leq \mathrm{J}^{1}\left(\gamma_{1}^{1}, \ldots, \gamma_{\mathrm{K}-1}^{1}, \gamma_{\mathrm{K}}^{1} ; \gamma_{1}^{2}, \ldots, \gamma_{\mathrm{K}-1}^{2}, \gamma_{\mathrm{K}}^{2^{*}}\right) \\
& \mathrm{J}^{2}\left(\gamma_{1}^{1}, \ldots, \gamma_{\mathrm{K}-1}^{1}, \gamma_{\mathrm{K}}^{1 *} ; \gamma_{1}^{2}, \ldots, \gamma_{\mathrm{K}-1}^{2}, \gamma_{\mathrm{K}}^{2^{*}}\right) \leq \mathrm{J}^{2}\left(\gamma_{1}^{1}, \ldots, \gamma_{\mathrm{K}-1}^{1}, \gamma_{\mathrm{K}}^{1 *} ; \gamma_{1}^{2}, \ldots, \gamma_{\mathrm{K}-1}^{2}, \gamma_{\mathrm{K}}^{2}\right)
\end{aligned}
$$

At stage $K-1$

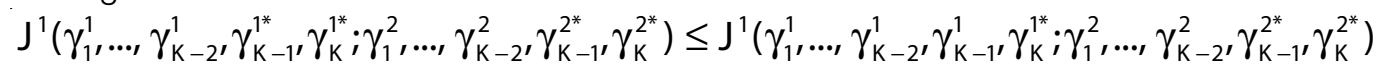

$$
\begin{aligned}
& \mathrm{J}^{2}\left(\gamma_{1}^{1}, \ldots, \gamma_{\mathrm{K}-2}^{1}, \gamma_{\mathrm{K}-1}^{1 *}, \gamma_{\mathrm{K}}^{1^{*}} ; \gamma_{1}^{2}, \ldots, \gamma_{\mathrm{K}-2}^{2}, \gamma_{\mathrm{K}-1}^{2^{*}} \gamma_{\mathrm{K}}^{2^{*}}\right) \leq \mathrm{J}^{2}\left(\gamma_{1}^{1}, \ldots, \gamma_{\mathrm{k}-2}^{1}, \gamma_{\mathrm{K}-1}^{1 *}, \gamma_{\mathrm{K}}^{1^{*}} ; \gamma_{1}^{2}, \ldots, \gamma_{\mathrm{K}-2}^{2}, \gamma_{\mathrm{K}-1}^{2}, \gamma_{\mathrm{K}}^{2^{*}}\right)
\end{aligned}
$$

\section{At stage 1}

$\mathrm{J}^{1}\left(\gamma^{1 *}, \gamma^{2^{*}}\right) \leq \mathrm{J}^{1}\left(\gamma_{1}^{1}, \gamma_{2}^{1 *}, \ldots \gamma_{\mathrm{k}}^{1^{*}} ; \gamma^{2^{*}}\right)$

$\mathrm{J}^{2}\left(\gamma^{1^{*}}, \gamma^{2^{*}}\right) \leq \mathrm{J}^{2}\left(\gamma^{1^{*}} ; \gamma_{1}^{2}, \gamma_{2}^{2^{*}}, \ldots \gamma_{\mathrm{K}}^{2^{*}}\right)$

For the purpose of the Stackelberg Equilibrium Solution, the following definitions will be required where $\mathbf{P} 1$ is the leader and $\mathbf{P} 2$ the follower. 
Definition 5. The set

$$
\mathrm{R}^{2}\left(\gamma^{1}\right)=\left\{\xi \in \Gamma^{2}: \mathrm{J}^{2}\left(\gamma^{1}, \xi\right) \leq \mathrm{J}^{2}\left(\gamma^{1}, \gamma^{2}\right), \forall \gamma^{2} \in \Gamma^{2}\right\}
$$

is the rational reaction set of $\mathbf{P} 2$ to the strategy $\gamma^{1} \in \Gamma^{1}$ of $\mathbf{P} 1$.

Remark 1. If the rational reaction set of the follower is a singleton for every announced strategy of the leader, then there exists a mapping $T^{2}=\Gamma^{1} \rightarrow \Gamma^{2}$, such that $\gamma^{2} \in \mathrm{R}^{2}\left(\gamma^{1}\right)$ implies $\gamma^{2}=T^{2} \gamma^{1}$. In words, the optimal response of the follower is unique for every strategy of the leader. The property of the rational reaction set is sufficient to ensure that the leader never does worse in the "Stackelberg game" than in the associated "Nash game". Economists are unaware of this restriction of the definition.

Definition 6. A strategy $\gamma^{\star \star} \in \Gamma^{1}$ is called a Stackelberg equilibrium strategy for the leader if

$$
J^{1}\left(\gamma^{1^{*}}, T^{2} \gamma^{1^{*}}\right)=\min _{\gamma^{1} \in \Gamma^{1}} J^{1}\left(\gamma^{1}, T^{2} \gamma^{1}\right)
$$

Let the above quantity be denoted by $\mathrm{J}^{1^{*}}$ which is the Stackelberg cost of the leader. It is not only the secured equilibrium cost for the leader but it is the cost level that is actually attained. For the follower, we have

Definition 7. Let $\gamma^{1 *} \in \Gamma^{1}$ be a Stackelberg equilibrium strategy for the leader. Any element $\gamma^{2^{*}} \in \mathrm{R}^{2}\left(\gamma^{1^{*}}\right)$ is an optimal strategy for the follower that is in equilibrium with $\gamma^{1^{*}}$. The pair $\left\{\gamma^{1^{*}}, \gamma^{2^{*}}\right\}$ is a Stackelberg solution for the game and the pair $\left\{J^{1}\left(\gamma^{1^{*}}, \gamma^{2^{*}}\right), J^{2}\left(\gamma^{1^{*}}, \gamma^{2^{*}}\right)\right\}$ is the corresponding Stackelberg equilibrium outcome.

For appreciating the notion of time consistency, the following notation and definitions are required. Following $\mathrm{Ba}$ ar and Jan Olsder (1995), we abuse notation by using the denoting the discrete time period by the continuous time period. Thus, $[0, T]=\{1, \ldots, K\}$. Let

$$
\mathrm{D}(\Gamma ;[0, \mathrm{~T}] ; \mathrm{N})
$$

denote a two-person dynamic game where is the product strategy space, $[0, T]$ is the decision interval and $N$ stands for the Nash solution concept. Also, let

$$
\gamma_{[s, t]} \in \Gamma_{[s, t]} \gamma_{[s, t]}^{\mathrm{i}} \in \Gamma_{[s, t]}^{\mathrm{i}}
$$

denote the truncations of $\gamma \in \Gamma$ and $\gamma^{\mathrm{i}} \in \Gamma^{\mathrm{i}}$, respectively, to the time period $[\mathrm{s}, \mathrm{t}] \subset[0, T]$, and let

$$
D_{[s, t]}^{\beta} \equiv D\left(\left\{\gamma \in \Gamma: \gamma_{[0, s)}=\beta_{[0, s)} \gamma_{(s, T]}=\beta_{(s, T]} \gamma_{[s, t]} \in \Gamma_{[s, t]}\right\} ;[0, T] ; N\right)
$$

denote a version of $\mathrm{D}(\Gamma ;[0, T] ; \mathrm{N})$ where the strategies of both players $i$ in the intervals $[0, s)$ and $(t, T]$ are fixed as $\beta_{[0,5),}^{i} \beta_{(s, T]}^{i}$. In that case, we have

Definition 8. A pair of strategies $\left\{\gamma^{1^{*}}, \gamma^{2^{*}}\right\}$ solving the dynamic game $\mathrm{D}(\Gamma ;[0, \mathrm{~T}] ; \mathrm{N})$ is said to be weakly time consistent (WTC) if its truncation to the interval $[\mathrm{s}, T], \gamma_{[\mathrm{S}, \mathrm{T}]}^{*}$, is the solution of the truncated game $\mathrm{D}_{[\mathrm{S}, \mathrm{T}}^{\gamma^{*}}$, for all $s \in(0, T]$. If a solution $\gamma^{*} \in \Gamma$ is not WTC, it is time inconsistent.

There is no basis at $k$ for cheating at future stages only if the past actions are consistent with the original solution. 


\section{The Results}

A fundamental role is played in the theory of differential games by the notion of 'representations of strategies along trajectories'. It enables the construction of equivalence classes of equal open-loop value strategies in the general class of closed-loop strategies. The procedure is as follows. First determine the set $\Gamma^{G}$ of all elements of $\Gamma$ which are strategies that depend only on the initial state $x_{1}$ and the discrete time parameter $k . \Gamma^{G}$ is the class of all permissible open-loop controls in $\Gamma$. Now, let $\gamma^{*}=\left\{\gamma_{\mathrm{k}}^{*}\left(\mathrm{x}_{1}\right), \mathrm{k} \in \mathrm{K}\right\}$ be a chosen element of $\Gamma^{\mathrm{G}}$ which generates, by substitution into the state equation, a unique trajectory $\left\{x_{k^{\prime}}^{*}, k \in K\right\}$. Then consider all elements $\gamma=\left\{\gamma_{k}(),. k \in K\right\}$ of $\Gamma^{G}$ with the properties

(i) $\quad \gamma$ generates the same trajectory as $\gamma^{*}$ and

(ii) $\quad \gamma_{k}\left(x_{k}^{*}, x_{k-1}^{*}, \ldots, x_{2}^{*}, x_{1}\right) \equiv \gamma_{k}^{*}\left(x_{1}\right), k \in K$.

The subset of $\Gamma$ thus constructed constitutes an equivalence class of representations all of which have the same open-loop value. If this procedure is carried out for every element of $\Gamma^{G}$ the construction of all equivalence classes of $\Gamma$ can be completed.

The following result (Başar and Olsder, 1995) which pertains to the information patterns of Definition 2 has been proved in Correa (1999).

Proposition 1. Under dynamic information, the Stackelberg solution is also a Nash equilibrium solution.

In economics, the problem is posed as a choice between optimality and time-consistency. Bellman's principle of optimality leads to time-consistent solutions. However, in models in which expectations are forward-looking, the timeconsistent solutions are suboptimal. The dynamic programming solution yields a higher cost than the ex ante, timeinconsistent optimal solution. In terms of the present discussion it can be clarified that the information pattern implicitly assumed is the MPS or the FB pattern. The sub optimality of the time-consistent policy arises because no account is taken of the effect of the control sequence after the first period. In other words,

Proposition 2. Under the MPS and FB information structures, the feedback Stackelberg equilibrium solution is time-inconsistent.

Proof. Suppose the players were operating under the FB information pattern. The identical argument carries over to the MPS information structure. At stage $k, \mathbf{P} 1$, say, decides to recommit. By virtue of the definition, it must be the case that both parties have reneged on an equilibrium path earlier. Her inducement to do so must be

$$
\begin{aligned}
& J^{1}\left(\gamma_{1}^{1^{*}}, \ldots, \gamma_{\mathrm{k}-1}^{1^{*}}, \gamma_{\mathrm{k}}^{1^{*}}, \gamma_{\mathrm{k}+1}^{1^{*}}, \ldots, \gamma_{\mathrm{k}}^{1^{*}} ; \gamma_{1}^{2^{*}}, \ldots, \gamma_{\mathrm{k}-1}^{2^{*}}, \gamma_{\mathrm{k}}^{2^{*}}, \gamma_{\mathrm{k}+1}^{2^{*}}, \ldots, \gamma_{\mathrm{k}}^{2^{*}}\right) \\
& \geq J^{1}\left(\gamma_{1}^{1^{*}}, \ldots, \gamma_{\mathrm{k}-1}^{1^{*}}, \tilde{\gamma}_{\mathrm{k}}^{1}, \gamma_{\mathrm{k}+1^{1 *}}^{1}, \ldots, \gamma_{\mathrm{k}}^{1^{*}} ; \gamma_{1}^{2^{*}}, \ldots, \gamma_{\mathrm{k}-1}^{2^{*}}, \gamma_{\mathrm{k}}^{2^{*}}, \gamma_{\mathrm{k}+1^{2}}^{2^{*}}, \ldots, \gamma_{\mathrm{k}}^{2^{*}}\right)
\end{aligned}
$$

where $\gamma_{k}^{i}=\psi\left(x_{k}\right), i=1,2$, is the feedback strategy under the FB information pattern. Like earlier movements away from decided paths that have been successful, $\mathbf{P} 2$ cannot be worse off by this new strategy. $\mathbf{P} 1$ 's action determines movement into a new equivalence class with initial condition $\left(\mathrm{x}_{1}, \tilde{\mathrm{x}}_{\mathrm{k}+1}\right)$. We can proceed to construct the set of strategies which only depend on this new initial state and the time periods that follow. This would be the class of 
feedback controls. An element would be $\left\{\tilde{\gamma}=\psi\left(\tilde{\mathrm{x}}_{\mathrm{k}+1}\right)\right\}$ which generates by substitution into the state evolution equation a unique trajectory $\tilde{\mathbf{x}}_{\mathrm{k}+2}$. We can proceed to construct an equivalence class of representations all of which have the same value $\tilde{\gamma}$.

Under the CLPS and MPS information patterns, on the other hand, the discussion can be summarized in the following statement.

Proposition 3. Under the OL and CLPS information structures, the feedback Stackelberg equilibrium solution is both time-consistent and optimal.

Proof. Consider a FSE $\left\{\gamma^{1^{*}}, \gamma^{2^{*}}\right\}$. At stage $k, \mathbf{P} 1$, the leader, decides to renege on this solution. As earlier, it must be the case that for some strategy $\tilde{\gamma}_{k^{\prime}}^{1}$

$$
\begin{aligned}
& J^{1}\left(\gamma_{1}^{1^{*}}, \ldots, \gamma_{\mathrm{k}-1}^{1^{*}}, \gamma_{\mathrm{k}}^{1^{*}}, \gamma_{\mathrm{k}+1}^{1^{*}}, \ldots, \gamma_{\mathrm{k}}^{1^{*}} ; \gamma_{1}^{2^{*}}, \ldots, \gamma_{\mathrm{k}-1}^{2^{*}}, \gamma_{\mathrm{k}}^{2^{*}}, \gamma_{\mathrm{k}+1}^{2^{*}}, \ldots, \gamma_{\mathrm{k}}^{2^{*}}\right) \\
& \geq J^{1}\left(\gamma_{1}^{1^{*}}, \ldots, \gamma_{\mathrm{k}-1}^{1^{*}}, \tilde{\gamma}_{\mathrm{k}}^{1}, \gamma_{\mathrm{k}+1}^{1 *}, \ldots, \gamma_{\mathrm{k}}^{1^{*}} ; \gamma_{1}^{2^{*}}, \ldots, \gamma_{\mathrm{k}-1}^{2^{*}} \gamma_{\mathrm{k}}^{2^{*}}, \gamma_{\mathrm{k}+1}^{2^{*}}, \ldots, \gamma_{\mathrm{k}}^{2^{*}}\right)
\end{aligned}
$$

The defection under CLPS or MPS implies entry into an information equivalence class where

$$
\gamma_{k}\left(\tilde{x}_{k}, \tilde{x}_{k-1}, \ldots, \tilde{x}_{2}, x_{1}\right) \equiv \tilde{\gamma}_{k}\left(x_{1}\right), k \in K
$$

Accordingly, the evolution of the state till stage $k$ is given by

$$
\tilde{\mathrm{x}}_{\mathrm{k}+1}=\mathrm{f}_{\mathrm{k}}\left(\tilde{\mathrm{x}}_{\mathrm{k}}, \tilde{\mathrm{u}}_{\mathrm{k}}^{1}, \tilde{\mathrm{u}}_{\mathrm{k}}^{2}\right), \tilde{\mathrm{x}}_{1}=\mathrm{x}_{1} \text {. }
$$

It turns out, therefore, that for agent $\mathbf{P} 1$, the leader, either

or

$$
\begin{aligned}
& J^{1}\left(\gamma_{1}^{1^{*}}, \ldots, \gamma_{k}^{1^{*}}, \gamma_{k+1}^{1^{*}}, \ldots, \gamma_{k}^{1 *} ; \gamma_{1}^{2^{*}}, \ldots, \gamma_{k}^{2^{*}}, \gamma_{k+1}^{2^{*}}, \ldots, \gamma_{k}^{2^{*}}\right) \\
& \geq J^{1}\left(\tilde{\gamma}_{1}^{1}, \ldots, \tilde{\gamma}_{k}^{1} \tilde{\gamma}_{k+1}^{1}, \ldots, \tilde{\gamma}_{k}^{1} ; \tilde{\gamma}_{1}^{2}, \ldots, \tilde{\gamma}_{k}^{2}, \tilde{\gamma}_{k+1}^{2}, \ldots \tilde{\gamma}_{k}^{2}\right) \\
& J^{1}\left(\gamma_{1}^{1 *}, \ldots, \gamma_{k}^{1^{*}}, \gamma_{k+1}^{1^{*}}, \ldots, \gamma_{k}^{1^{*}} ; \gamma_{1}^{2^{*}}, \ldots, \gamma_{k}^{2^{*}}, \gamma_{k+1}^{2^{*}}, \ldots, \gamma_{k}^{2^{*}}\right) \\
& \leq J^{!}\left(\tilde{\gamma}_{!}^{1}, \ldots, \tilde{\gamma}_{k}^{1}, \tilde{\gamma}_{k+1}^{1}, \ldots, \tilde{\gamma}_{k}^{1} ; \tilde{\gamma}_{1}^{2}, \ldots, \tilde{\gamma}_{k}^{2}, \tilde{\gamma}_{k+1}^{2}, \ldots, \gamma_{k}^{2}\right)
\end{aligned}
$$

If the first inequality holds, the assumption that the solution was a game-equilibrium is violated. If the second inequality is valid, the inducement to deviate from the Nash or Stackelberg solution evaporates. Analogous reasoning holds for the other player (the follower). In other words, for all $\left\{\gamma_{\mathrm{k}}^{\mathrm{i}} \in \Gamma_{\mathrm{k}}^{\mathrm{i}} ; \mathrm{i}=1,2 ; \mathrm{k} \in \mathrm{K}\right\}$,

$$
J^{i}\left(\gamma_{1}^{1^{*}}, \ldots, \gamma_{k}^{1^{*}} ; \gamma_{1}^{2^{*}}, \ldots, \gamma_{k}^{2^{*}}\right) \leq J^{i}\left(\gamma_{1}^{1}, \ldots, \gamma_{k}^{1} ; \gamma_{1}^{2}, \ldots, \gamma_{k}^{2}\right)
$$

The FNE or FSE solution is both optimal and time-consistent.

The notion of optimality used in the results is entirely information-specific and different from the concept in Economics according to which the notion is information-invariant. A definition that would be recognized by an economist is as follows: 
Definition 9. A pair of strategies $\left\{\gamma^{1^{*}}, \gamma^{2^{*}}\right\}$ with $\gamma^{i^{*}} \in \Gamma^{i}, i=1,2$, is said to constitute an Optimal Solution if, and only if, the following inequalities are satisfied for all $\gamma^{i} \in \Gamma^{i}, i=1,2$

$$
J^{i *} \cong J^{i}\left(\gamma^{1^{*}}, \gamma^{2^{*}}\right) \leq J^{i}\left(\gamma^{1}, \gamma^{2}\right)
$$

Thus, the DSGE model leads to an optimum optimorum. The result is unsurprising given the assumption that all agents are assumed to operate with one model. Even if possessed of different models and boundedly rational, agents will through rational learning come to cognize the only 'true' model.

\section{Conclusion}

The postulate of rational expectations is that agents use all information generated by the model. Our results, however, hinge on the assumptions concerning the choice of information sets. If the economy is in a general equilibrium with nice properties, rational expectations delivers the familiar result that neither the government nor the private sector is better off reneging on the social equilibrium. If, on the other hand, the economy is in an unemployment equilibrium and agents suffer from, say, imperfect recall, both parties are better off 'cheating' on the solution and moving to a dominating solution set. The fundamental construct here is that outcome space consists of classes of solutions.

\section{References}

1. Başar, T., Olsder, G.J. (1995). Dynamic Noncooperative Game Theory. Academic Press, London.

2. Correa, R. (1999). Dynamic Consistency under Private Information, Control and Cybernetics, 28 (2), 225-232.

3. Dow, S., Klaes, M., Montagnol, A. (2009). Risk and Uncertainty in Central Bank signals; An analysis of monetary policy committee minutes, Metroeconomica, 60 (4), 584-618.

4. Fischbocher, L.B.U. (2010). Taking the initiative. What motivates leaders? Thurgau Institute of economics and Department of Economics University of Konstantz Research Paper Series No.61.

5. Kirman, A. (2010). Walras' Unfortunate Legacy, GREQAM, Université d'Aix-Marseille, Document de travail, No. 2010-58

6. Tubaro, P. (2009). Agent-based Economics: A Methodological Appraisal, Université Paris X Nanterre, Economix Document de Travail, No. 2009-42

Romar Correa is the Reserve Bank of India Professor of Monetary Economics at the University of Mumbai. He works on the interface between micro and macro in non neoclassical frameworks. He has published extensively on the subject and is on the editorial board of journals devoted to the theme. A product of the 'Bombay School of Economics' he exploits the history of economic thought, mathematics, sociology, for the purposes on hand. 